\title{
Pattern of adverse events of antiepileptic drugs: results of the aESCAPE study in Poland
}

\author{
Barbara Chmielewskaํ, Krystyna Lis², Konrad Rejdak ${ }^{1}$, Marcin Balcerzak ${ }^{3}$, Barbara Steinborn ${ }^{4}$
}

\author{
1Department of Neurology, Medical University of Lublin, Lublin, Poland \\ 2NEURO-MED Medical Center, Katowice, Poland \\ 3UCB Pharma, Warsaw, Poland \\ ${ }^{4}$ Department of Developmental Neurology, Poznan University of Medical Sciences, \\ Poznan, Poland
}

Submitted: 30 June 2011

Accepted: 4 September 2011

Arch Med Sci 2013; 9, 5: 858-864

DOI: 10.5114/aoms.2013.38679

Copyright @ 2013 Termedia \& Banach

\begin{abstract}
Introduction: The Adverse Event Scale in Patients With Epilepsy (aESCAPE) European study (NCT00394927) explored and analyzed adverse events (AEs) and reasons for modifying treatment in patients treated with newer and older antiepileptic drugs (AEDs) used in monotherapy or polytherapy. The present analysis concerns the results of patients recruited in Poland.

Material and methods: Multicentre, international, observational, cross-sectional study investigating AEs in patients with epilepsy (aged $\geq 4$ years), on stable $A E D$ treatment with one or two $A E D(s)$ for $\geq 3$ months, using standardized questionnaires completed by a physician during a single study visit.

Results: Out of 309 patients, $24.6 \%$ were treated exclusively with newer AED(s) in monotherapy or in combination, while $75.4 \%$ were treated with older AED(s) or a combination of older and newer $\mathrm{AED}(\mathrm{s}) .60 .8 \%$ were on monotherapy, and $39.9 \%$ on polytherapy. In general, $73.8 \%$ of patients reported $\geq 1 \mathrm{AE}(\mathrm{s})$. There were no significant differences in the frequency of reported AEs in compared groups. The most common were disturbances in cognitive function (40.5\%), psychological problems (36.2\%), and sedation (32.7\%). Some AEs were found to be more specific for particular types and treatment regimens. Changes in treatment or dose during the study visit occurred in $22.3 \%$ of the patients, mainly due to lack of efficacy (10.7\%), AEs (5.2\%) or absence of seizures (4.5\%).

Conclusions: A detailed structured interview revealed high frequency of AEs in patients treated with AEDs. The main reasons for treatment modifications at the study visit were lack of efficacy, adverse events and absence of seizures.
\end{abstract}

Key words: adverse events, antiepileptic drugs, epilepsy, Poland.

\section{Introduction}

The chronic nature of epilepsy and long-term pharmacotherapy focus concern on patients' safety. Since 1990, the number of approved antiepileptic drugs (AEDs) has been increasing progressively, and the development of a newer generation of AEDs continues. Monotherapy is preferred over polytherapy and, whenever possible, therapy adapted to the needs of individual patients is chosen $[1,2]$. Although newer AEDs are not considered more efficacious than conventional anticonvulsants, they may be better tolerated [3-7].

\author{
Corresponding author: \\ Prof. Barbara Steinborn \\ Department \\ of Developmental Neurology \\ Poznan University \\ of Medical Sciences \\ 49 Przybyszewskiego St \\ 60-335 Poznan, Poland \\ Phone: +48 618691255 \\ Fax: +48 618691553 \\ E-mail: bstein@ump.edu.pl
}


Tolerability profile is an important factor determining the choice of appropriate AED for an individual patient. However, it is known that the spontaneous reporting of adverse events (AEs) leads to underestimation of their diversity and frequency [8] Evaluation of antiepileptic drug therapy with standardized questionnaires, including lists of AEs, increases their detection and allows one to understand individual patients' problems and optimize their therapy [9-12].

This study (adverse Event Scale in Patients with Epilepsy Study - aESCAPE; NCT00394927) aimed to assess the rate and distribution of neurological and systemic AEs related to antiepileptic treatment with older and newer AEDs, as well as to monotherapy and polytherapy. The overall results of the aESCAPE study, performed in 6 European countries, have been described by Cramer et al. [13]. This report presents the results of analysis reflecting the prevalence and types of AEs associated with AEDs use, and reasons for treatment modification in the population of Polish patients with epilepsy enrolled in the aESCAPE study.

\section{Material and methods}

The aESCAPE (NCT00394927) study was an observational, cross-sectional, multicentre, multicounty, surveillance, in-label study carried out in the Czech Republic, Germany, Italy, Poland, Romania and Spain in 2007 [13]. All patients provided written informed consent before the study. The study was approved by the Independent Ethics Committee. In Poland, 15 sites participated in patient recruitment. The methodology of the study was described in detail by Cramer et al. [13].

The AE experiences of patients with epilepsy were evaluated with a structured questionnaire developed for the longitudinal Veterans Administration Cooperative Studies (Neurological and Systemic Adverse Event Rating Scales [N\&SAERS]) [14], translated from English to Polish. Physicians completed the questionnaire during one study visit, on the basis of one structured interview, neurological and physical assessment during the visit, and medical history files. The primary outcome variable of the study was the percentage of patients reporting $\geq 1$ AE(s) (based on N\&SAERS). Secondary outcome variables were: prevalence of each type of $A E$, patient and epilepsy characteristics, and reasons for treatment modifications.

Patients aged $>4$ years, diagnosed with epilepsy, with stable treatment with one or two AED(s) for $\geq 3$ months, could participate in the survey. Severe or uncontrolled symptomatic chronic illness concomitant to epilepsy excluded patients from the study. The choice of antiepileptic medication was not determined by the study protocol. Drugs used were classified into two categories: 1) newer AEDs: gabapentin (GBP), lamotrigine (LTG), levetiracetam (LEV), oxcarbazepine (OXC), tiagabine (TGB), topiramate (TPM), or any combination of these; 2 ) older AEDs: carbamazepine (CBZ), clobazam (CLB), clonazepam (CZP), phenobarbital (PB), phenytoin (PHT), valproate (VPA) and any combination of two older AEDs or a combination of one older and one newer AED. All AEDs had to be used in accordance with the Polish marketing authorization valid at the time of the study.

Because of the explanatory and cross-sectional nature of the aESCAPE study (NCT00394927), a formal sample size calculation was not performed. For the analysis, two ways of patient grouping were defined, depending on the type and number of concomitant AEDs used: patients receiving only newer AEDs vs. those receiving older AEDs (including a combination of one older and one newer AED); and patients on monotherapy versus those receiving polytherapy. Percentages of patients who reported $\geq 1 \mathrm{AE}(\mathrm{s})$, as described in N\&SAERS, are presented. A logistic regression model on the presence of $\geq 1 \mathrm{AE}(\mathrm{s})$ in terms of type of treatment (polytherapy vs. monotherapy, newer vs. older AEDs) was performed. Descriptive statistics for the decision to modify treatment at the study visit are presented. Logistic regression models on the proportion of patients modifying treatment were performed, including a number of explanatory variables: type of treatment (polytherapy vs. monotherapy, newer vs. older AEDs), presence vs. absence of AEs, generalized vs. not generalized seizures, and time since the latest seizure ( $\geq 1$ vs. $<1$ year ago).

\section{Results}

\section{Patients and antiepileptic drugs}

In Poland, 309 patients were recruited. They constituted $30.9 \%$ of all patients participating in the aESCAPE study ( $n=1019)$. Demographic and clinical characteristics were largely similar in the analyzed groups. Partial-onset seizures (POS) were diagnosed in $73.5 \%$ of the patients and $24.3 \%$ of the patients were diagnosed with primary generalized seizures (PGS) (Table I). 61\% of the patients $(n=188)$ were on monotherapy, among whom $70 \%(n=132)$ were treated with an older AED. Out of the patients on a polytherapy regimen ( $n=121), 75.2 \%$ were on a combination of older and newer AEDs, $8.2 \%$ used a combination of two older AEDs, and $16.5 \%$ used two newer AEDs (Table I). Overall, in the Polish population, patients were on a stable dose regimen for a median of 10 months at the time of the study. The median time since the last seizure was 183 days. Polytherapy regimen was associated with a 2.5 times shorter period since the last seizure than the period generally observed in the overall Polish population (Table I). 
Table I. Demographics, epilepsy and treatment characteristics

\begin{tabular}{|c|c|c|c|c|c|}
\hline Characteristics & Overall $(n=309)$ & Newer $(n=76)$ & Oldera $^{2}(n=233)$ & Mono $(n=188)$ & Poly $(n=121)$ \\
\hline \multicolumn{6}{|l|}{ Genderd $^{d}$} \\
\hline Female, $n(\%)$ & $157(50.8)$ & $45(59.2)$ & $112(48.1)$ & $95(50.5)$ & $62(51.2)$ \\
\hline Male, $n(\%)$ & $151(48.9)$ & $30(39.5)$ & $121(51.9)$ & $92(48.9)$ & $59(48.8)$ \\
\hline \multicolumn{6}{|l|}{ Age [years] } \\
\hline Mean \pm SD & $23.92 \pm 16.72$ & $21.53 \pm 13.35$ & $24.70 \pm 17.63$ & $21.89 \pm 16.66$ & $27.02 \pm 16.41$ \\
\hline Range & $4-82$ & $4-73$ & $4-82$ & $4-79$ & $4-82$ \\
\hline \multicolumn{6}{|c|}{ Age at onset [years] } \\
\hline Mean \pm SD & $13.76 \pm 13.46$ & $12.57 \pm 10.69$ & $14.15 \pm 14.24$ & $14.34 \pm 13.67$ & $12.88 \pm 13.12$ \\
\hline Range & $1-78$ & $1-78$ & $1-78$ & $1-72$ & $1-78$ \\
\hline \multicolumn{6}{|c|}{ Duration since last seizure [days] b,e } \\
\hline Median $_{(\mathrm{Q} 1-\mathrm{Q} 3)}$ & $183_{(30-517)}$ & $183_{(32-365)}$ & $183_{(25-525)}$ & $274_{(84-660)}$ & $66_{(10-216)}$ \\
\hline \multicolumn{6}{|c|}{ Duration since last treatment modification [months] ${ }^{\mathrm{b}}$} \\
\hline Median $_{(\mathrm{Q} 1-\mathrm{Q} 3)}$ & $10_{(5-19)}$ & $8_{(5-15)}$ & $11_{(5-22)}$ & $11_{(6-22)}$ & $8_{(5-18)}$ \\
\hline \multicolumn{6}{|c|}{ Type of seizurec,f, $n$ (\%) } \\
\hline POS only & $227(73.5)$ & $58(76.3)$ & $169(72.5)$ & $129(68.6)$ & $98(81.0)$ \\
\hline PGS only & $75(24.3)$ & $17(22.4)$ & $58(24.9)$ & $55(29.3)$ & $20(16.5)$ \\
\hline POS \& PGS & $3(1.0)$ & - & $3(1.3)$ & - & $3(2.5)$ \\
\hline \multicolumn{6}{|c|}{ AED by medication, $n(\%)$} \\
\hline$\overline{C B Z}$ & $86(27.8)$ & - & $86(26.9)$ & $50(26.6)$ & $36(29.8)$ \\
\hline CLN & $3(1.0)$ & - & $3(1.3)$ & - & $3(2.5)$ \\
\hline GBP & $13(4.2)$ & $7(9.2)$ & $6(2.6)$ & $3(1.6)$ & $10(8.3)$ \\
\hline$\overline{\mathrm{LTG}}$ & $63(20.4)$ & $29(38.2)$ & 34 (14.6) & 20 (10.6) & $43(35.5)$ \\
\hline LEV & $16(5.2)$ & $6(7.9)$ & $10(4.3)$ & $2(1.1)$ & 14 (11.6) \\
\hline $\mathrm{OXC}$ & 49 (15.9) & $33(43.4)$ & $16(6.9)$ & $22(22.3)$ & $27(22.3)$ \\
\hline $\mathrm{PB}$ & $2(0.6)$ & - & $2(0.9)$ & - & $2(1.7)$ \\
\hline$\overline{\mathrm{PHT}}$ & $3(1.0)$ & - & $3(1.3)$ & $2(1.1)$ & $1(0.8)$ \\
\hline TGB & $10(3.2)$ & $5(6.6)$ & $5(2.1)$ & $1(0.5)$ & $9(7.4)$ \\
\hline TPM & $36(11.7)$ & $16(21.1)$ & $20(8.6)$ & $8(4.3)$ & $28(23.1)$ \\
\hline VPA & $149(48.2)$ & - & $149(48.2)$ & $80(42.6)$ & $69(57.0)$ \\
\hline \multicolumn{6}{|l|}{ AED by group, $n(\%)$} \\
\hline 1 older & $132(42.7)$ & - & $132(56.6)$ & $132(70.2)$ & - \\
\hline 1 newer & 56 (18.1) & $56(73.7)$ & - & $56(29.8)$ & - \\
\hline 2 older & $10(3.2)$ & - & $10(4.3)$ & - & $10(8.3)$ \\
\hline 2 newer & $20(6.5)$ & $20(26.3)$ & - & - & $20(16.5)$ \\
\hline 1 older +1 newer & $91(29.4)$ & - & $91(39.0)$ & - & $91(75.2)$ \\
\hline
\end{tabular}

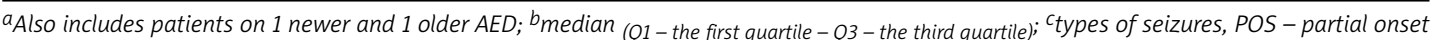
seizures including simple partial seizures, complex partial seizures, partial onset seizures with secondary generalization alone and in combinations; PGS - primarily generalized seizures including absence, myoclonic, clonic, tonic-clonic, atonic seizures alone and in combination; ${ }^{g}$ gender data were missing for one patient, ${ }^{e}$ age and age at epilepsy onset data were missing for 3 patients, $f$ type of seizure data were missing for 4 patients

Almost half of the patients were treated with VPA (48.2\%). Commonly used AEDs in monotherapy were: VPA (42.6\%), CBZ (26.6\%), OXC (11.7\%), LTG (10.6\%), and in combination therapy: LTG + VPA (20.7\%), OXC + VPA (11.6\%), TPM + VPA (9.1\%).

\section{Prevalence and types of adverse events}

In the Polish population, $73.8 \%$ of patients reported $\geq 1 \mathrm{AE}(\mathrm{s})$ (66\% reported $\geq 1$ neurological $\mathrm{AE}(\mathrm{s})$, $41.4 \%$ reported $\geq 1$ systemic $A E(s)$ ) (Figure 1 ). 
A slightly higher percentage of Polish patients on older $\mathrm{AED}(\mathrm{s})$ reported $\geq 1 \mathrm{AE}(\mathrm{s})$ in comparison with patients on newer $\mathrm{AED}(\mathrm{s}) \mathrm{OR}=0.59,95 \% \mathrm{Cl}$ : $0.31-$ $1.14, p=0.1182$ ) (Figure 1). The percentage of patients reporting $\geq 1 \mathrm{AE}(\mathrm{s})$ was similar in monotherapy $(71.3 \%)$ and polytherapy $(77.7 \%)$ groups $(\mathrm{OR}=1.15,95 \% \mathrm{Cl}: 0.61-2.15, p=0.6744)$.

Overall, the most commonly reported AEs (> 10\% patients) were: disturbances in cognitive function, psychological problems, sedation, gain or loss of weight, headache, tremor, gastrointestinal complaints and dizziness (Table II). The following AEs were reported more frequently by patients on older $A E D(s)$ or a combination of older and newer $A E D(s)$ than by patients on newer AED(s): disturbances in cognitive function, sedation and gastrointestinal problems (Table II). Polytherapy, as compared with monotherapy, was to a greater extent associated with cognitive function disturbance, sedation, tremor, ataxia, dysarthria, diplopia and gastrointestinal problems (Table II).

\section{Treatment modifications}

The decision regarding treatment modification was made during the study visit for $22.3 \%$ of the patients $(15.9 \%$ - dose change and $6.5 \%$ - AED change). Treatment modification was introduced less often for patients on newer AEDs (13.2\%) or for patients on monotherapy (17.0\%) than for those

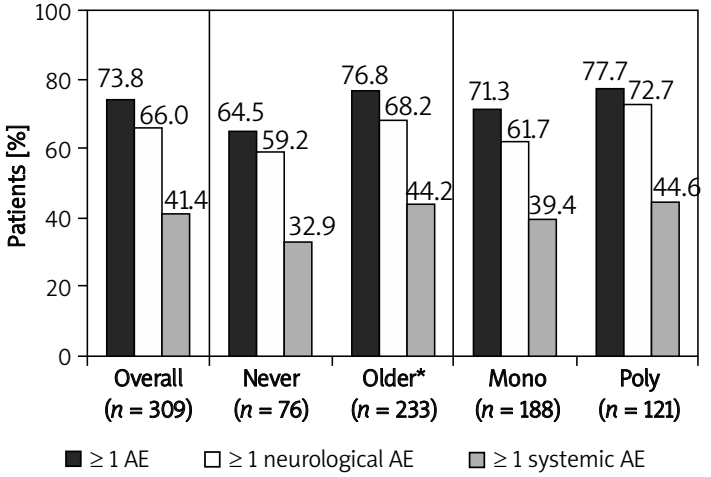

Figure 1. Patients reporting $\geq 1 \mathrm{AE}, \geq 1$ neurological $A E$, $\geq 1$ systemic $A E$

${ }^{*}$ Also includes patients on 1 newer and 1 older AED

on older AEDs (25.3\%) or for patients on polytherapy (30.6\%), respectively (Figure 2). The main reasons for changing AEDs were lack of efficacy (50\%), or AEs (30\%). The main reasons for changing drug doses were lack of efficacy $(46.9 \%)$, absence of seizures (24.5\%), or AEs (20.5\%) (data not shown).

During the study visit, the treatment was more likely to be modified for patients on polytherapy than for patients on monotherapy $(\mathrm{OR}=1.98,95 \% \mathrm{Cl}$ : $1.07-3.66, p=0.029)$. Logistic regression analysis showed that the likelihood of physicians modifying treatment for patients who had been seizure-free for $\geq 1$ year (in $15.0 \%$ of cases) was smaller than in the case of patients who had had a seizure $<1$ year

Table II. Incidence (\%) of AEs reported by $\geq 5 \%$ of patients in any group

\begin{tabular}{|c|c|c|c|c|c|}
\hline Parameter & Overall $(n=309)$ & Newer $(n=76)$ & $\operatorname{Older}^{\mathrm{a}}(n=233)$ & Mono $(n=188)$ & Poly $(n=121)$ \\
\hline \multicolumn{6}{|l|}{ Neurological AE [\%] } \\
\hline $\begin{array}{l}\text { Cognitive function } \\
\text { disturbance }\end{array}$ & 40.5 & 30.3 & $43.8^{\star \star}$ & 31.4 & $54.5^{\text {***}}$ \\
\hline $\begin{array}{l}\text { Psychological } \\
\text { problems }\end{array}$ & 36.2 & 31.6 & 37.8 & 32.4 & 42.1 \\
\hline Sedation & 32.7 & 22.4 & $36.1^{\star *}$ & 28.2 & $39.7^{\star \star}$ \\
\hline Headache & 18.4 & 12.0 & 13.6 & 14.4 & 12.4 \\
\hline Tremor & 13.6 & 9.2 & 15.0 & 8.5 & $21.5^{\star \star \star}$ \\
\hline Dizziness & 10.4 & 6.6 & 11.6 & 8.0 & 14.0 \\
\hline Ataxia & 4.5 & 2.6 & 5.2 & 2.7 & $7.4^{\star \star}$ \\
\hline Dysarthria & 3.6 & 6.6 & 2.6 & 0.5 & $8.3^{\star \star \star}$ \\
\hline Diplopia & 3.2 & 6.6 & 2.1 & 1.6 & $5.8^{\star \star}$ \\
\hline \multicolumn{6}{|l|}{ Systemic AE [\%] } \\
\hline Gain or loss of weight & 19.4 & 15.8 & 20.6 & 19.7 & 19.0 \\
\hline GI problems & 12.0 & 2.6 & $15.0^{\star \star * \star}$ & 8.5 & $15.4^{\star \star}$ \\
\hline Arthralgia & 8.4 & 5.3 & 9.4 & 10.1 & 5.8 \\
\hline $\begin{array}{l}\text { Changes in hair } \\
\text { quantity and texture }\end{array}$ & 7.8 & 5.3 & 8.6 & 6.9 & 9.1 \\
\hline Lack of menstrual cyclec & 5.5 & 9.2 & 4.3 & 4.8 & 6.6 \\
\hline
\end{tabular}

${ }^{a}$ Also includes patients on 1 newer and 1 older AED; b depression, tension/agitation, anger/hostility, vigor/excitability, fatigue/apathy, confusion/thought disorder; ${ }^{c}$ if applicable; ${ }^{* *} p<0.05,{ }^{* *} p<0.01$ 


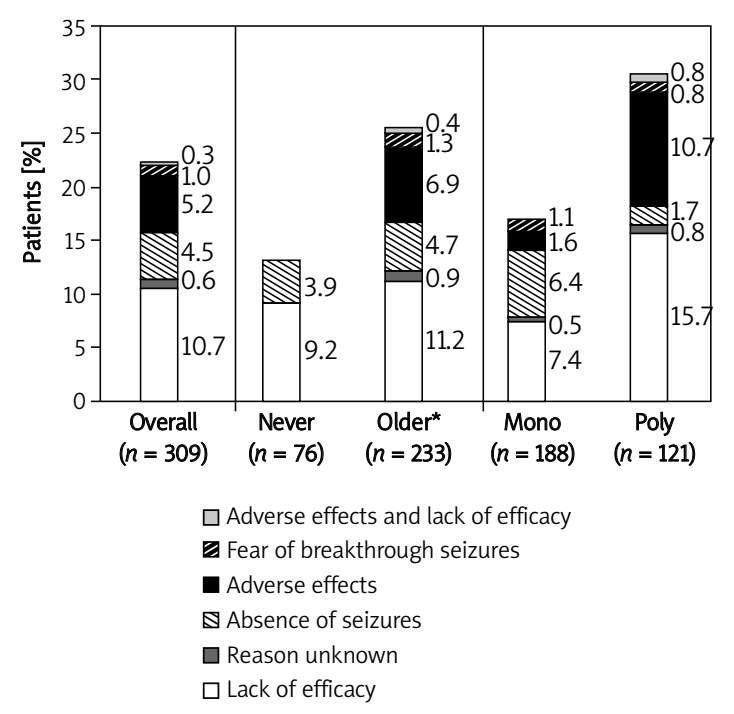

Figure 2. Reason for treatment modification in patients whose treatment was changed during the study visit by AED group

${ }^{*} A$ lso includes patients on 1 newer and 1 older AED

ago (in $25.8 \%$ of cases) $(\mathrm{OR}=0.53,95 \% \mathrm{Cl}$ : $0.27-1.06$, $p=0.07)$. Presence of AEs ( $\geq 1 \mathrm{AE}(\mathrm{s})$ vs. no $\mathrm{AE}$, $\mathrm{OR}=0.71,95 \% \mathrm{Cl}: 0.36-1.42, p>0.3)$ and treatment with newer AEDs (never vs. older AEDs, OR $=0.64$, $95 \% \mathrm{Cl}$ : 0.29-1.38, $p>0.2$ ) did not affect the likelihood of treatment modification.

\section{Discussion}

Development of the systematic screening and rating scale of AEs to assess AED safety has facilitated the identification of AEs [9-12], which, by leading to treatment modification, could improve tolerability. Standardized questionnaires for patients and physicians seem to act as detailed tools for precise detection and monitoring of undesired effects of AEDs [10, 14]. According to Carreno et al. [9], the use of a questionnaire, as compared with spontaneous reporting, has increased reporting of AEs about twofold. Canevini et al. [15] indicated that the use of the Adverse Event Profile questionnaire, as compared with spontaneous reporting, has increased reporting of AEs 13 times. As also shown in previous studies $[9,12]$, about $2 / 3$ of patients reported AEs after having been provided with a checklist, which is consistent with the results obtained in the subpopulation of Polish patients enrolled in the aESCAPE study.

Newer AEDs are used when initial therapy with older AEDs has failed. Many Polish physicians use VPA and CBZ (for almost $1 / 2$ and $1 / 4$ of patients, respectively) on the basis of extensive clinical experience, and because they are devoted to VPA as a drug having a broad spectrum of efficacy [16]. In this analysis, newer AEDs were used by $55 \%$ of patients, mainly in combinations with older AEDs (Table I).
In general, newer AEDs appear to be better tolerated than older AEDs [3-7]. The overall results of the aESCAPE study reported by Cramer et al. [13] indicated that patients taking newer AEDs report $\geq 1 \mathrm{AE}(\mathrm{s})$ much less frequently than patients on older AEDs $(\mathrm{OR}=0.64, p=0.008)$. In the Polish subpopulation the percentage of patients reporting $\geq 1 \mathrm{AE}(\mathrm{s})$ was slightly higher in the group using at least 1 older AED than in the group using newer $\mathrm{AED}(\mathrm{s})$ (Figure 1) $(\mathrm{OR}=0.59, p=0.118)$, which is in accordance with previous observations [3-9] and with the overall results of the aESCAPE study [13]; however, statistical significance was not reached, possibly due to the small sample size.

Previous studies suggested that polytherapy increases the probability of AEs $[4,12]$ and reduction or elimination of polytherapy may be the way to minimize the number of AEs [17]. Polish patients using two AEDs reported $\geq 1 \mathrm{AE}(\mathrm{s})$ slightly more frequently than those on monotherapy (Figure 1) $(\mathrm{OR}=1.15, p=0.674)$. One of the limitations of our study is that the population of patients on polytherapy was composed of persons using no more than two AEDs. In fact, dual therapy may not reflect common problems of polytherapy in which many patients take three or more AEDs. According to the results of a previous national study, $11.8 \%$ of patients used $\geq 3$ AEDs [18]. In the Polish subpopulation of the aESCAPE study, most of the patients on monotherapy (70.2\%) and almost all patients on polytherapy (83.5\%) were using older AED(s). A recent cross-sectional study which was performed in Italy, and in which AE profiles of refractory patients on mono- or polytherapy were compared, did not show a difference in the burden of AEs, even though the most prevalent medications in both groups were newer AEDs [15].

We observed that cognitive dysfunction and sedation were reported much more frequently by patients treated with older AEDs or on polytherapy than by patients treated with newer AEDs or on monotherapy. These AEs were the most frequent reasons for patient complaints in previous studies $[12,19]$. It should be noted that in a previous study [9], subjective complaints, such as cognitive and mood problems and tiredness, were more frequently reported via checklist than spontaneously, which emphasizes the importance of a systematic approach for detection of these AEs. It is worth noting that patients on polytherapy reported neurotoxicity-related AEs, such as tremor, dysarthria, diplopia and ataxia, more frequently than patients on monotherapy. These AEs are difficult to avoid when using polytherapy [20], which was also reflected in the study by Carreno et al. [9]: patients using polytherapy reported tremor, speech difficulties, diplopia and blurred vision, walking difficulties and stumbling much more frequently than patients on monotherapy. 
Physicians in Poland decided to modify treatment for $22.3 \%$ of patients. This is similar to the results obtained in the overall aESCAPE population (22.8\%) [13] and those reported by Carreno et al. (26.4\%) [9]. While adequate seizure control in making clinical decisions was very important, lack of efficacy was the main reason for treatment modifications in the Polish population. Lack of efficacy was the most important reason for both AED change and dose modification. Additionally, time since latest seizure shorter than 1 year was found to be a predictor of treatment modification $(\mathrm{OR}=$ $0.53, p=0.07$ ), which is consistent with the findings of Cramer et al. [13] concerning the overall aESCAPE population $(\mathrm{OR}=0.39, p<0.001)$. Indeed, achieving a seizure-free state may improve life quality to the level observed in the general population [21]. Lack of efficacy was a reason for almost $50 \%$ of treatment modifications; AEs accounted for about $23 \%$. This is similar to the results obtained by Carreno et al. [9], where about $60 \%$ of treatment changes were carried out due to inadequate seizure control and $23 \%$ due to AEs. The overall results of the aESCAPE study have shown that lack of efficacy and AEs accounted for $1 / 3$ of treatment modifications [13]. It should be noted that median time since the latest seizure was shorter in the Polish subpopulation (6.5 months) than median time observed in the overall aESCAPE population (7.7 months), which may partially explain higher attention of Polish doctors' to treatment efficacy issues. Moreover, the strongest predictor of treatment modification was a polytherapy regimen $(\mathrm{OR}=1.98, p=0.029)$ where patients had shorter time since the latest seizure (Table I), which may also account for the observed frequent treatment modifications attributed to the lack of efficacy. Thus, treatment tolerability has not been found to be a factor influencing treatment modification in Polish patients $(\mathrm{OR}=0.71, p>0.3)$. However, in the European aESCAPE study, a trend to modify treatment in the case of AE occurrence was observed ( $O R=1.48$, $p=0.055$ ) [13]. Interestingly, as regards the Polish patients treated exclusively with newer $\operatorname{AED}(\mathrm{s})$, no treatment adjustments were suggested during the study visit due to AEs. However, due to the non-randomized and cross-sectional character of the study, we cannot attribute it to newer AED(s) use, since other factors (e.g. severity of AEs) may also be important. Absence of seizures was a frequent reason $(20.3 \%)$ for treatment modification. Recent results indicate that such an intervention results in the improvement of life quality [22].

Limitations to these analyses include the nonrandomized, cross-sectional study design. Since the treatment was not randomly assigned, baseline differences in disease state and possibly other factors, which could be related to the primary outcome variable, were more likely to occur. Observations made at one point in time do not provide information about a sequence of events (which is particularly important as regards studying chronic illness) and follow-up. In order to avoid overestimation of newer AEDs' usefulness and to account for heterogeneity of the patient population with different treatment regimens, the patients treated with a combination of one newer and one older AED were classified in the group of "older AEDs". Analyses are also limited by studying only the Polish patients enrolled in the overall aESCAPE study. The small number of patients taking newer AEDs did not allow for adequate comparisons for some AEDs. In addition, the fact that almost half of the patients were taking VPA alone or in combination overweighed analyses toward that AED.

Although the Polish subpopulation analysis results revealed a high number of patient complaints in the course of epilepsy treatment, only a small part of them required treatment adjustment due to $A E(s)$ occurrence. Treatment modifications were mainly related to lack of efficacy and concerned mostly patients on polytherapy. Results from the Polish subpopulation of the aESCAPE study suggest that more resistant clinical forms of epilepsy occur as the reasons of treatment adjustment more frequently than the AED tolerability issues. This study demonstrated that by using the N\&SAERS, physicians can quickly and easily determine the scope of AEDs' adverse effects during routine visits $[13,14,19]$. The N\&SAERS provides a structured approach to monitoring a wide spectrum of possible AEs in the course of epilepsy treatment.

\section{Acknowledgments}

This study was sponsored by UCB, who were involved in designing and conducting the study, collecting, managing, and analyzing the data, as well as preparing and reviewing the manuscript. The authors would like to thank the members of the aESCAPE Polish Study Group for their valued contribution to the study: Jolanta Bielicka-Cymerman, Barbara Błaszczyk, Barbara Chmielewska, Jacek Gawłowicz, Tomasz Kmieć, Małgorzata Krause, Krystyna Lis, Maria Mazurkiewicz-Bełdzińska, Anatol Mickielewicz, Konrad Rejdak, Andrzej Rysz, Barbara Seńkowska, Barbara Steinborn, Marek Szatanik, and Marzanna Wołk. We thank Joyce Cramer (Yale University, USA), Iga Bechyne-Put (UCB Pharma, Poland), Sarah Lu (UCB Inc, US), Robert Chan, Svetlana Dimova, Benjamin Duncan, Charlotte Nortvedt, Francoise Tonner, and Laurent Turet (all from UCB Pharma S.A., Belgium), who provided critical review and editing support.

\section{References}

1. Marson AG, Kadir ZA, Hutton JL, Chadwick DW. The new antiepileptic drugs: a systematic review of their efficacy and tolerability. Epilepsia 1997; 38: 859-80. 
2. Schmidt D, Gram L. Monotherapy versus polytherapy in epilepsy. A reappraisal. CNS Drugs 1995; 3: 194-208.

3. Perucca E, Meador KJ. Adverse events of antiepileptic drugs. Acta Neurol Scand Suppl 2005; 112: 30-5.

4. Malphrus AD, Wilfong AA. Use of the newer antiepileptic drugs in pediatric epilepsies. Curr Treat Options Neurol 2007; 9: 256-67.

5. Cramer JA, Fisher R, Ben-Menachem E, French J, Mattson RH. New antiepileptic drugs: comparison of key clinical trials. Epilepsia 1999; 40: 590-600.

6. Cramer JA, Ben Menachem E, French J. Review of treatment options for refractory epilepsy: new medications and vagal nerve stimulation. Epilepsy Res 2001; 47: 17-25.

7. Kanner AM, Balabanov AJ. The use of monotherapy in patients with epilepsy: an appraisal of the new antiepileptic drugs. Curr Neurol Neurosci Rep 2005; 5: 322-8.

8. Gilliam F. What we don't learn from clinical trials in epilepsy. Epilepsia 2003; 44 (Suppl. 7): 51-4.

9. Carreno M, Gil-Nagel A, Sanchez JC, et al. Strategies to detect adverse effects of antiepileptic drugs in clinical practice. Epilepsy Behav 2008; 13: 178-83.

10. Gilliam FG, Fessler AJ, Baker G, Vahle V, Carter J, Attarian H. Systematic screening allows reduction of adverse antiepileptic drug effects: a randomized trial. Neurology 2004; 62: 23-7.

11. Mei PA, Montenegro MA, Guerreiro MM, Guerreiro CA. Pharmacovigilance in epileptic patients using antiepileptic drugs. Arq Neuropsiquiatr 2006; 64: 198-201.

12. Uijl SG, Uiterwaal CS, Aldenkamp AP, et al. A crosssectional study of subjective complaints in patients with epilepsy who seem to be well-controlled with anti-epileptic drugs. Seizure 2006; 15: 242-8.

13. Cramer JA, Steinborn B, Striano P, et al. A multicenter, cross-sectional, non-interventional surveillance study to evaluate the adverse effects of antiepileptic drug treatment in patients with epilepsy. Acta Neurol Scand 2011; 124: 13-21.

14. Cramer JA, Smith DB, Mattson RH, Delgado Escueta AV, Collins JF. A method of quantification for the evaluation of antiepileptic drug therapy. Neurology 1983; 33 (3 Suppl. 1): 26-37.

15. Canevini MP, De Sarro G, Galimberti CA, et al. Relationship between adverse effects of antiepileptic drugs, number of coprescribed drugs, and drug load in a large cohort of consecutive patients with drug-refractory epilepsy. Epilepsia 2010; 51: 797-804.

16. Aldenkamp A, Vigevano F, Arzimanoglou A, Covanis A. Role of valproate across the ages. Treatment of epilepsy in adults. Acta Neurol Scand Suppl 2006; 184: 1-13.

17. St. Louis EK. Minimizing AED adverse effects: improving quality of life in the interictal state in epilepsy care. Curr Neuropharmacol 2009; 7: 106-14.

18. Majkowski J, Majkowska-Zwolinska B. Demographic, social and medical characteristics of 1019 epileptic patients. a prospective, multi-centre study of the costs of epilepsy in Poland in 2006. Epileptologia 2007; 15: 119-39.

19. Carpay JA, Aldenkamp AP, van Donselaar CA. Complaints associated with the use of antiepileptic drugs: results from a community-based study. Seizure 2005; 14: 198-206.

20. Deckers CL. Overtreatment in adults with epilepsy. Epilepsy Res 2002; 52: 43-52.

21. Leidy NK, Elixhauser A, Vickrey B, Means E, Willian MK. Seizure frequency and the health-related quality of life of adults with epilepsy. Neurology 1999; 53: 162-6.

22. Uijl SG, Uiterwaal CS, Aldenkamp AP, et al. Adjustment of treatment increases quality of life in patients with epilepsy: a randomized controlled pragmatic trial. Eur J Neurol 2009; 16: 1173-7. 